\title{
The necessary and sufficient conditions for the solutions of elliptic problems
}

\author{
G.M. Gharib* \\ Mathematics Department, College of Science, and Information Technology, \\ Zarqa University, Jordan.
}

\begin{abstract}
This is the necessary and sufficient conditions to the regularity of solution of elliptic problems on non smooth domains in $R^{3}$. I study a boundary value problem for elliptic partial differential equation. I study the regularity of solution to the problem in non smooth domain. I obtain the necessary and sufficient conditions of the problem to belong to $C_{m+2+\alpha}$.
\end{abstract}

Keywords: Elliptic problem, Non smooth domain, regularity of solution.

AMS(MOS) Subject Classification: 35A20, 35B65, 46B20.

\section{Introduction}

The regularities of the solutions on nonsmooth domains are typically described in terms of usual Sobolev spaces and the asymptotic expansions where the solutions are decomposited into regular and singular parts (see [1-12]).

In engineering applications many problems in $R^{3}$ are characterized by partial differential equations with piecewise analytic data such as nonsmooth domains, abruptly changes of types of boundary conditions, piecewise analytic coefficients and boudary conditions, etc., for instance, the physical domains of structral mechanical problems often have edges and vertices, interfaces between di?erent materials and material cracks [13-15]. The solutions of these problems have strong sin- gularities at the edges and vertices and around the cracks, which make the conventional numerical approximation extremely difficult and inefficient. Hence comprehensive study on the regularity of the solutions of elliptic problems in $R^{3}$ with piecewise analytic data is of great significance not only for theoretical reasons but also for the desigh of effective computations and the optimal convergence of numerical method for these problems [16-20].

\footnotetext{
^ E-mail: Gharibmusa@gmail.com
} 
These regularity results are important and useful for the regularity theory for elliptic problems on nonsmooth domains and for solving these problems by conventional numerical approaches. But these results do not characterize sufficiently the class of solutions of the problems in applications. The conformal mapping and boundary value problems for harmonic functions; see, Lubuma [21], Maz'ya [22] or Maz'ya [23] was the earliest impetus. And the physical applications; examples can be found in [24], [25] and other standard monographs see also [26] and [27]. Also those problems play a role in numerical analysis, particulary in the study of the accuracy of finite element and finite difference approximation, acceleration of convergence, general convergence analysis, subtraction of singularities and other numerical techniques [28].

\section{Theorem}

Consider the initial Dirichlet problem

$$
\begin{gathered}
\triangle u-u_{t}=f(x, y, t), \quad(x, y, t) \in \Omega, \\
u(x, y, 0)=K(x, y), \\
\left.u\right|_{\Gamma_{0}}=\phi(y, t) \quad 0 n \Gamma_{0} \times J \\
\left.u\right|_{\Gamma_{0}}=\psi(r, t) \quad 0 n \Gamma_{\omega} \times J \\
\text { where } \quad r=\left(x^{2}+y^{2}\right)^{\frac{1}{2}} .
\end{gathered}
$$

The necessary and sufficient conditions for the solutions of problem (1) - (2) to belong to $C_{m+2+\alpha}(\Omega)$ are:

(i) $f(x, y, t) \in C_{m+\alpha}(\bar{\Omega}), \quad \phi(y, t) \in C_{m+2+\alpha}\left(\Gamma_{0} \times J\right)$ and $\psi_{\omega}(r, t) \in C_{m+2+\alpha}\left(\Gamma_{1} \times J\right)$

$$
\text { (ii) } \phi(y, 0)=K(0, y) \quad \phi(0, t)=\psi(0, t)
$$

(iii) $\quad \psi_{\omega}^{(\gamma q)}=(-1)^{\gamma} \phi_{0}^{\gamma q}+\sum_{p=2}^{\gamma q}\left\{\sum_{j=1}^{\frac{p}{2}}\left[\sum_{k=0}^{\frac{p}{2}-j}(-1)^{k}\left(\begin{array}{l}k+j-1 \\ j-1\end{array}\right)\left(\begin{array}{l}\gamma q \\ p\end{array}\right) \cdot \cos ^{\gamma q-p} \omega \sin ^{p} \omega P_{j-1}^{(p-2 k-2 j, \gamma q-p+2 k)}-\right.\right.$

$$
\left.\left.(-1)^{\gamma+k}\left(\begin{array}{c}
\left(\frac{p}{j}\right] \\
j
\end{array}\right) \cdot \phi_{j}^{\gamma q-2 k}\right]\right\}, \quad \gamma=1,2, \ldots,\left[\frac{m+2+\alpha}{q}\right] .
$$

The necessity of conditions (i) and (ii) is obvious. To prove the necessity of condition (iii), we notice that 


$$
u_{\omega}^{(\gamma q)}(r, t)=\sum_{p=0}^{\gamma q}\left(\begin{array}{c}
\gamma q \\
p
\end{array}\right) \cos ^{\gamma q-p} \omega \sin ^{p} \omega u^{(p, \gamma q-p)}(x, y, t),
$$

and form equation (5) we obtain

$$
\begin{gathered}
u_{\omega}^{(\gamma q)}(r, t)=\cos ^{\gamma q} \omega u^{(0, \gamma q)}(x, y, t)+\left(\begin{array}{l}
\gamma q \\
1
\end{array}\right) \cos ^{\gamma q-1} \omega \sin \omega u^{(1, \gamma q-1)}(x, y, t)+ \\
\sum_{p=2}^{\gamma q}\left(\begin{array}{c}
\gamma q \\
p
\end{array}\right) \cos ^{\gamma q-p} \omega \sin ^{p} \omega\left[\sum_{j=1}^{\frac{p}{2}} \sum_{k=0}^{\frac{p}{2}-j}(-1)^{k}\left(\begin{array}{l}
k+j-1 \\
j-1
\end{array}\right) f_{j-1}^{(p-2 k-2 j, \gamma q-p+2 k)}(x, y, t)-\right. \\
\left.\sum_{k=0}^{\frac{p}{2}}(-1)^{k}\left(\begin{array}{c}
\frac{p}{2} \\
k
\end{array}\right) u_{k}^{\left(p-2 \frac{p}{2}-2 j, \gamma q-p+2 k\right)}(x, y, t)\right]=L f-\sum_{p=0}^{\frac{\gamma q}{2}}\left(\begin{array}{c}
\gamma q \\
2 p
\end{array}\right) \cos ^{\gamma q-2 p} \omega \sin ^{2 p} \omega \sum_{k=0}^{\frac{p}{2}}(-1)^{k}\left(\begin{array}{l}
\frac{p}{2} \\
k
\end{array}\right) u_{k}^{(0, \gamma q-2 k)} \\
-\sum_{p=1}^{\frac{\gamma q}{2}}(-1)^{p-1}\left(\begin{array}{l}
\gamma q \\
2 p-1
\end{array}\right) \cos ^{\gamma q-2 p+1} \omega \sin ^{2 p-1} \omega \sum_{k=0}^{\frac{p}{2}}(-1)^{k}\left(\begin{array}{l}
\frac{p}{2} \\
k
\end{array}\right) u_{k}^{(1, \gamma q-2 k)} \\
\text { where } \quad \psi_{\omega}^{\gamma q}=L f-\sum_{k=0}^{\frac{p}{2}}(-1)^{\gamma+k}\left(\begin{array}{l}
\frac{p}{2} \\
k
\end{array}\right) \phi_{k}^{(0, \gamma q-2 k)} \\
(\cos \omega+i \sin \omega)^{\gamma q}=(-1)^{\gamma} \text { and } \\
L f=\sum_{p=2}^{\gamma q} \sum_{j=1}^{\frac{p}{2}} \sum_{k=0}^{\frac{p}{2}-j}(-1)^{k}\left(\begin{array}{l}
\gamma q \\
p
\end{array}\right)\left(\begin{array}{l}
k+j-1 \\
j-1
\end{array}\right) \cos ^{\gamma q-p} \omega \sin ^{p} \omega f_{j-1}^{(p-2 k-2 j, \gamma q-p+2 k)}
\end{gathered}
$$

\section{Proof}

The sufficiency of the conditions depends on a constructed function (c.f. lemma 2). This function is constructed to remove the discontinuities at the boundary $\Gamma_{0}$. These discontinuities are appeared where we continue the solution by symmetry across the boundary $\Gamma_{0}$. The new boundary functions and right hand side of the equation are then shown to satisfy the compatibility condition $\left(7^{\prime}\right)$. By repeating this process we can extend the domain until the angle is $\pi$, with the boundary function belonging to $C_{m+2+\alpha}$.

We first prove

\section{Lemma 1.}

By the function $f(x, y, t) \in C_{m+\alpha}(\bar{\Omega})$, we can construct functions $f_{p}^{\star}(x, y, t), P=0,1, \cdots, m$, defined on the whole plane and having the properties.

I. $f_{p}^{\star}(0, y, t)=\frac{\partial^{p} f(0, y, t)}{\partial x^{p}}, \quad 0 \leq y \leq a, \quad t \geq 0$,

II. $\quad f_{p}^{\star}(-x, y, t)=f_{p}^{\star}(x, y, t)$, 
III. $f_{p}^{\star}(x, y, t) \in C_{m-p+\alpha}(\bar{\Omega})$,

IV. $\quad x^{i} \frac{\partial^{j} f_{p}^{\star}(x, y, t)}{\partial x^{j_{1}} \partial y^{j_{2}} \partial t^{j_{3}}} \in C_{m-p-j+i+\alpha}(\bar{\Omega}), \quad i \geq j$,

V. $\quad \frac{\partial^{2 j_{1}+j_{3}} f_{p}^{\star}(0, y, t)}{\partial x^{2 j_{1}-2 i} \partial y^{2 i} \partial t^{j_{3}}}=A_{j}^{p} \frac{\partial^{2 j_{1}+j_{3}} f_{p}^{\star}(0, y, t)}{\partial x^{2 j_{1}} \partial t^{j_{3}}}, \quad 0 \leq y \leq a, \quad A_{j}^{p}>0$.

\section{Proof}

For simplicity we prove the lemma only for $p=0$, the same proof can be used for $p=1,2, \cdots, m$. Consider an averaging kernel $K(s) \in C_{\infty}, \quad-\infty<s<\infty$, with the properties

(a) $\quad K(s) \geq 0$ if $|s|<1, \quad K(s)=0$ if $|s| \geq 1$,

(b) $K(s)$ is an even function,

(c) $\quad \int_{-\infty}^{\infty} K(s) d s=1$.

Setting $f(x, y, t)=0$ if $(x, y, t) \notin \bar{\Omega}$, we define $f^{\star}(x, y, t)=f_{0}^{\star}(x, y, t)$ as follows

$$
f^{\star}(x, y, t)=\int_{-\infty}^{\infty} K(s) f(0, y-x s, t-x s) d s .
$$

Setting $\eta=y-x s, \quad \xi=t-x s$, using the mean value theorem, we obtain from (3)

$f^{\star}(x, y, t)=\int_{-1}^{1} K(s) f(0, y-x s, t-x s) d s=f\left(0, y-x s_{0}, t-x s_{0}\right) \int_{-1}^{1} K(s) d s=f\left(0, y-x s_{0}, t-x s_{0}\right)$,

where $s_{0} \in(-1,1)$. Letting $x$ tends to zero we obtain property I.

Changing $s$ to $-s$ in (3), and noting that $K(s)$ is even, we obtain property II.

It is clear that $f^{\star}(x, y, t) \in C_{\alpha}(\bar{\Omega})$, for

$$
\begin{aligned}
& \left|f^{\star}\left(x_{2}, y_{2}, t_{2}\right)-f^{\star}\left(x_{1}, y_{1}, t_{1}\right)\right| \leq \int_{-1}^{1} K(s)\left|f\left(0, y_{2}-x_{2} s, t_{2}-x_{2} s\right)-f\left(0, y_{1}-x_{1} s, t_{1}-x_{1} s\right)\right| d s \\
& \leq B\left\{\left[\left(y_{2}-x_{2} s\right)-\left(y_{1}-x_{1} s\right)\right]^{2}+\left[\left(t_{2}-x_{2} s\right)-\left(t_{1}-x_{1} s\right)\right]^{2}\right\}^{\frac{\alpha}{2}} . \\
& \text { But } \quad(a-s b)^{2} \leq\left(2\left(a^{2}+s^{2} b^{2}\right) \leq 2\left(a^{2}+b^{2}\right), \quad|s| \leq 1 .\right.
\end{aligned}
$$

Then

$$
\left(\left|f^{\star}\left(x_{2}, y_{2}, t_{2}\right)-f^{\star}\left(x_{1}, y_{1}, t_{1}\right)\right| \leq M\left[\left(x_{2}-x_{1}\right)^{2}+\left(y_{2}-y_{1}\right)^{2}+\left(t_{2}-t_{1}\right)^{2}\right]^{\frac{\alpha}{2}}, .\right.
$$

where

$$
\int_{-1}^{1} K(s) d s=1, \text { and for any } K \leq m,
$$

$$
\begin{gathered}
\frac{\partial^{k} f^{\star}(x, y, t)}{\partial x^{k_{1}} \partial y^{k_{2}} \partial t^{k_{3}}}=\int_{-\infty}^{\infty} \frac{\partial^{k_{1}+k_{2}} K(s)}{\partial x^{k_{1}} \partial y^{k_{2}}} \frac{\partial^{k_{3}}}{\partial t^{k_{3}}} f(0, y-s x, t-s x) d s \\
=\int_{-\infty}^{\infty} \frac{\partial^{k_{1}} K(s)}{\partial x^{k_{1}}}\left[\frac{\partial^{k_{2}+k_{3}}}{\partial \eta^{k_{2}} \partial \xi^{k_{3}}} f(0, y-s x, t-s x)\right] d s
\end{gathered}
$$




$$
=\int_{-\infty}^{\infty} K(s)(-s)^{k_{1}} \sum_{i=0}^{k_{1}}\left({ }_{i}^{k_{1}}\right) \frac{\partial^{k_{1}}}{\partial \eta^{k_{1}-i} \partial \xi^{i}} \frac{\partial^{k_{2}+k_{3}}}{\partial \eta^{k_{2}} \partial \xi^{k_{3}}} f(0, y-s x, t-s x) d s,
$$

and this may be shown as before to belong to $C_{\alpha}(\bar{\Omega}$. This proves property III. Equation (5) may be written as

$$
\begin{gathered}
\frac{\partial^{k} f^{\star}(x, y, t)}{\partial x^{k_{1}} \partial y^{k_{2}} \partial t^{k_{3}}}=\int_{-\infty}^{\infty} K(s)(-s)^{k_{1}} \sum_{i=0}^{k_{1}}\left({ }_{i}^{k_{1}}\right) \frac{\partial^{k_{1}}}{\partial \eta^{k_{1}-i} \partial \xi^{i}}(-x)^{-\left(k_{2}+k_{3}\right)} \frac{\partial^{k_{2}+k_{3}}}{\partial s^{k_{2}+k_{3}}} f(0, \eta, \xi) d s \\
\int_{-\infty}^{\infty} K(s)(-s)^{k_{1}}(-x)^{-k} \frac{\partial^{k}}{\partial s^{k}} f(0, \eta, \xi) d s .
\end{gathered}
$$

Then

$$
x^{k} \frac{\partial^{k} f^{\star}(x, y, t)}{\partial x^{k_{1}} \partial y^{k_{2}} \partial t^{k_{3}}}=(-1)^{k_{2}+k_{3}} \int_{-\infty}^{\infty} K(s)(s)^{k_{1}} \frac{\partial^{k}}{\partial s^{k}} f(0, \eta, \xi) d s,
$$

which, after integrating by parts $k$ times, gives

$$
x^{k} \frac{\partial^{k} f^{\star}(x, y, t)}{\partial x^{k_{1}} \partial y^{k_{2}} \partial t^{k_{3}}}=(-1)^{k_{1}} \int_{-1}^{1} \frac{d^{k}}{d s^{k}}\left[K(s)(s)^{k_{1}}\right] f(0, \eta, \xi) d s,
$$

where

$$
\left[\frac{d^{k-n}}{d s^{k-n}}\left[K(s)(s)^{k_{1}}\right] \frac{\partial^{n-1}}{\partial s^{n-1}} f(0, \eta, \xi)\right]_{-1}^{1}=0, \quad n=1,2, \cdots, k, \quad k(s)=0, \quad|s| \geq 1 .
$$

As before this may be shown to belong to $C_{m+\alpha}(\bar{\Omega})$

i.e. $\quad x^{k} \frac{\partial^{k} f^{\star}(x, y, t)}{\partial x^{k_{1}} \partial y^{k_{2}} \partial t^{k_{3}}} \in C_{m+\alpha}(\bar{\Omega})$.

Property IV follows since

$$
\frac{\partial^{i-j}}{\partial x^{i_{1}} \partial y^{i_{2}} \partial t^{i_{3}}}\left(x^{i} \frac{\partial^{j} f^{\star}(x, y, t)}{\partial x^{j_{1}} \partial y^{j_{2}} \partial t^{j_{3}}}\right)=\sum_{k=j}^{i} \beta_{k} x^{k} \frac{\partial^{k} f^{\star}(x, y, t)}{\partial x^{k_{1}} \partial y^{k_{2}} \partial t^{k_{3}}},
$$

where $\quad x^{k} \frac{\partial^{k} f^{\star}(x, y, t)}{\partial x^{k_{1}} \partial y^{k_{2}} \partial t^{k_{3}}} \in C_{m+\alpha}(\bar{\Omega})$, then $\quad x^{i} \frac{\partial^{j} f_{p}^{\star}(x, y, t)}{\partial x^{j_{1}} \partial y^{j_{2}} \partial t^{j} j_{3}} \in C_{m-p-j+i+\alpha}(\bar{\Omega}), \quad i \geq j$

From (11) we obtain

$$
\begin{gathered}
\frac{\partial^{k} f^{\star}(x, y, t)}{\partial x^{k_{1}} \partial y^{k_{2}} \partial t^{k_{3}}}=\int_{-\infty}^{\infty}\left[K(s)(-s)^{k_{1}}\right]\left[\sum_{j=0}^{k}\left(\begin{array}{l}
k \\
j
\end{array}\right) \frac{\partial^{k} f(0, \eta, \xi)}{\partial \eta^{k-j} \partial \xi^{j}}\right] d s, \\
\therefore \quad \frac{\partial^{2 k_{1}+k_{3}} f^{\star}(x, y, t)}{\partial x^{2 k_{1}-2 i} \partial y^{2 i} \partial t^{k_{3}}}=\left[\sum_{j=0}^{k}\left(\begin{array}{c}
k \\
j
\end{array}\right) \frac{\partial^{k} f\left(0, y-x s_{0}, t-x s_{0}\right)}{\partial \eta^{k-j} \partial \xi^{j}}\right] \int_{-1}^{1}\left[K(s)(s)^{2 k_{1}-2 i}\right] d s \\
=\alpha_{k-i}\left[\sum_{j=0}^{k}\left(\begin{array}{l}
k \\
j
\end{array}\right) \frac{\partial^{k} f\left(0, y-x s_{0}, t-x s_{0}\right)}{\partial \eta^{k-j} \partial \xi^{j}}\right],
\end{gathered}
$$

where $s_{0} \in(-1,1)$ and, $\quad \alpha_{k}=\int_{-1}^{1}\left[K(s)(s)^{2 k}\right] d s>0$.

Letting $x$ tends to zero we obtain 


$$
\frac{\partial^{2 k_{1}+k_{3}} f^{\star}(0, y, t)}{\partial x^{2 k_{1}-2 i} \partial y^{2 i} \partial t^{k_{3}}}=\alpha_{k-i}\left[\sum_{j=0}^{k}\left(\begin{array}{l}
k \\
j
\end{array}\right) \frac{\partial^{k} f(0, y, t)}{\partial y^{k-j} \partial t^{j}}\right]
$$

put $i=0$, then

$$
\frac{\partial^{2 k_{1}+k_{3}} f^{\star}(0, y, t)}{\partial x^{2 k_{1}-2 i} \partial y^{2 i} \partial t^{k_{3}}}=A_{k}^{0} \frac{\partial^{2 k_{1}+k_{3}}}{\partial x^{2 k_{1}} \partial t^{k_{3}}} f(0, y, t) .
$$

From this, property $\mathrm{V}$ follows with $A_{k}^{0}=\frac{\alpha_{k-i}}{\alpha_{k}}, \quad 0<A_{k}^{0}<1$. Then the lemma is proved.

\section{Lemma 2.}

There exist functions $g_{p}(x, y, t) \in C_{m+2+\alpha}(\bar{\Omega}), \quad p=0,1, \ldots, m$ with the property that

$$
\begin{gathered}
\left(\triangle-\frac{\partial}{\partial t}\right) g_{p}=\frac{x^{p}}{p !} f^{(p, 0,0)}(0, y, t)+\sum_{j=0}^{n-1} \sum_{k=0}^{n-1-j} a_{k j}^{p} x^{p+2+2 k+2 j}\left\{\frac { \partial ^ { 2 k } } { \partial x ^ { 2 k } } \left[f^{(p, 0, j+1)}(0, y, t)-\right.\right. \\
\left.\left.f^{\star(p, 0, j+1)}(0, y, t)\right]\right\}+o\left(x^{m}\right),
\end{gathered}
$$

where $a_{k j}^{p}$ are some constants.

\section{Proof.}

We construct $g_{p}(x, y, t)$ as follows

$$
g_{p}(x, y, t)=\sum_{j=0}^{n} \sum_{k=0}^{n-j} a_{k j}^{p} x^{p+2+2 k+2 j} \frac{\partial^{2 k}}{\partial x^{2 k}}\left[f^{\star^{(p, 0, j)}}(x, y, t)\right]
$$

where $\quad a_{0 j}^{p}=\frac{1}{(p+2+2 j) !}$, and we fined the other coefficients $a_{k j}^{p}$ by induction

$$
\begin{aligned}
& \triangle g_{p}=\frac{x^{p}}{p !} f^{\star^{(p, 0,0)}}(x, y, t)+\frac{2 x^{p+1}}{(p+1) !} \frac{\partial}{\partial x} f^{\star^{(p, 0,0)}}(x, y, t)+\frac{x^{p+2}}{(p+2) !}\left[\frac{\partial^{2} f^{\star^{(p, 0,0)}}}{\partial x^{2}}+\frac{\partial^{2} f^{\star^{(p, 0,0)}}}{\partial y^{2}}\right] \\
& +a_{10}^{p}\left[(p+4)(p+3) x^{p+2} \frac{\partial^{2} f^{\star}(p, 0,0)}{\partial x^{2}}+\ldots\right]+\triangle \sum_{k=2}^{n} a_{k 0}^{p} x^{p+2+2 k} \frac{\partial^{2 k}}{\partial x^{2 k}}\left[f^{\star^{(p, 0,0)}}(x, y, t)\right]+ \\
& \frac{x^{p+2}}{(p+2) !} f^{\star^{(p, 0,0)}}(x, y, t)+\frac{2 x^{p+3}}{(p+3) !} \frac{\partial}{\partial x} f^{\star^{(p, 0,1)}}(x, y, t)+\frac{x^{p+4}}{(p+4) !}\left[\frac{\partial^{2} f^{\star^{(p, 0,1)}}}{\partial x^{2}}+\frac{\partial^{2} f^{\star^{(p, 0,1)}}}{\partial y^{2}}\right] \\
& +a_{11}^{p}\left\{(p+6)(p+5) x^{p+4} \frac{\partial^{2} f^{\star^{(p, 0,1)}}}{\partial x^{2}}+2(p+6) x^{p+5} \frac{\partial^{3} f^{\star^{(p, 0,1)}}}{\partial x^{3}}+x^{p+6}\left[\frac{\partial^{4} f^{\star^{(p, 0,1)}}}{\partial x^{4}}+\frac{\partial^{4} f^{\star^{(p, 0,1)}}}{\partial y^{4}}\right]\right\} \\
& +\triangle\left\{\sum_{k=2}^{n-1} a_{k 1}^{p} x^{p+4+2 k} \frac{\partial^{2 k}}{\partial x^{2 k}}\left[f^{\star}{ }^{(p, 0,1)}(x, y, t)\right]+\sum_{j=2}^{n} \sum_{k=0}^{n-j} a_{k j}^{p} x^{p+2+2 k+2 j} \frac{\partial^{2 k}}{\partial x^{2 k}}\left[f^{\star^{(p, 0, j)}}(x, y, t)\right]\right\} .
\end{aligned}
$$


Expanding the functions involved and using the properties of $f_{p}^{\star}(x, y, t)$ we obtain

$$
\begin{aligned}
& \triangle g_{p}=\frac{x^{p}}{p !}\left[f^{(p, 0,0)}(0, y, t)+\frac{x^{2}}{2 !} \frac{\partial^{2}}{\partial x^{2}} f^{(p, 0,0)}(0, y, t)+\cdots\right]+\frac{2 x^{p+1}}{(p+1) !}\left[\frac{x \partial^{2} f^{(p, 0,0)}(0, y, t)}{\partial x^{2}}+\cdots\right] \\
& +\frac{\left(1+A_{1}^{p}\right) x^{p+2}}{(p+2) !}\left[\frac{\partial^{2}}{\partial x^{2}} f^{(p, 0,0)}(0, y, t)+\cdots\right]+a_{10}^{p}\left[(p+4)(p+3) x^{p+2} \frac{\partial^{2} f^{(p, 0,0)}(0, y, t)}{\partial x^{2}}+\ldots\right] \\
& +\frac{x^{p+2}}{(p+2) !}\left[f^{(p, 0,1)}(0, y, t)+\frac{x^{2}}{2 !} \frac{\partial^{2}}{\partial x^{2}} f^{(p, 0,1)}(0, y, t)+\cdots\right]+\frac{2 x^{p+3}}{(p+3) !}\left[\frac{x \partial^{2} f^{(p, 0,1)}(0, y, t)}{\partial x^{2}}+\cdots\right] \\
& +\frac{\left(1+A_{1}^{p}\right) x^{p+4}}{(p+4) !}\left[\frac{\partial^{2}}{\partial x^{2}} f^{(p, 0,1)}(0, y, t)+\cdots\right]+a_{11}^{p}\left[(p+6)(p+5) x^{p+24} \frac{\partial^{2} f^{(p, 0,1)}(0, y, t)}{\partial x^{2}}+\ldots\right] \\
& +\triangle\left\{\sum_{k=2}^{n} a_{k 0}^{p} x^{p+2+2 k} \frac{\partial^{2 k}}{\partial x^{2 k}}\left[f^{(p, 0,0)}(x, y, t)\right]+\sum_{k=2}^{n-1} a_{k 1}^{p} x^{p+4+2 k} \frac{\partial^{2 k}}{\partial x^{2 k}}\left[f^{\star^{(p, 0,1)}}(x, y, t)\right]\right. \\
& \left.+\sum_{j=2}^{n} \sum_{k=0}^{n-j} a_{k j}^{p} x^{p+2+2 k+2 j} \frac{\partial^{2 k}}{\partial x^{2 k}}\left[f^{\star^{(p, 0, j)}}(x, y, t)\right]\right\} .
\end{aligned}
$$

We choose $a_{10}^{p}$ such that the coefficient of $x^{p+2} \frac{\partial^{2} f^{(p, 0,0)}(0, y, t)}{\partial x^{2}}$ vanishes, and then we choose $a_{11}^{p}$ from the relation

$$
\frac{1}{2 !(p+2) !}+\frac{2}{(p+3) !}+\frac{\left(1+A_{1}^{p}\right)}{(p+4) !}+(p+5)(p+6) a_{11}^{p}=a_{10}^{p} .
$$

Suppose now that $a_{10}^{p}, a_{20}^{p}, \cdots, a_{(s-1) 0}^{p}$ have been already found, such that the coefficients of $x^{p+2 k} \frac{\partial^{2} f^{(p, 0,0)}(0, y, t)}{\partial x^{2}}, k=1,2, \cdots, s-1$ vanish and then we choose $a_{k, j+1}^{p}, \quad k=1,2,3, \cdots, s-2-$ $j \& j=0,1,2, \cdots, s-2$ such that

$$
\begin{gathered}
\frac{1}{(2 k) !(p+2+2 j) !}+\frac{2}{(2 k-1) !(p+3+2 j) !}+\frac{\left(1+A_{1}^{p}\right)}{(2 k-2) !(p+4) !}+ \\
\sum_{i=j}^{j+k-1}\left\{\left[\frac{(p+6+2 i)(p+5+2 i)}{(2 k+2 j-2 i-2) !}+\frac{2(p+6+2 i)}{(2 k--2 i-3) !}+\frac{\left(1+A_{1-j+2}^{p}\right)}{(2 k+2 j-2 i-4) !}\right] a_{i-j+1, j+1}^{p}\right\}=a_{k, j}^{p} .
\end{gathered}
$$

Then $\Delta g_{p}(x, y, t)$ may be written as

$$
\begin{gathered}
\Delta g_{p}(x, y, t)=\frac{x^{p}}{p !} f^{(p, 0,0)}(0, y, t)+\alpha_{s} x^{p+2 s} \frac{\partial^{2 s}}{\partial x^{2 s}} f^{(p, 0,0)}(0, y, t) \\
+\triangle \sum_{k=s}^{n} a_{k 0}^{p} x^{p+2+2 k} \frac{\partial^{2 k}}{\partial x^{2 k}}\left[f^{\star^{(p, 0,0)}}(x, y, t)\right]+\sum_{j=0}^{s-2} \sum_{k=0}^{s-2-j} a_{k j}^{p} x^{p+2+2 k+2 j} \frac{\partial^{2 k}}{\partial x^{2 k}}\left[f^{(p, 0, j+1)}(x, y, t)\right]
\end{gathered}
$$




$$
+\Delta \sum_{j=s}^{n} \sum_{k=0}^{n-j} a_{k j}^{p} x^{p+2+2 k+2 j} \frac{\partial^{2 k}}{\partial x^{2 k}}\left[f^{\star^{(p, 0, j)}}(x, y, t)\right]+O\left(x^{p-2+2 s}\right) .
$$

Choosing $a_{s 0}^{p}=\frac{-\alpha_{s}}{(p+2 s+2)(p+2 s+1)}$, the coefficient of $x^{p+2 s} \frac{\partial^{2 s}}{\partial x^{2 s}} f^{(p, 0,0)}$ will vanish and then choose $a_{k, j+1}^{p}$, where $k=1,2,3, \cdots, s-1-j \& j=0,1,2,3, \cdots, s-1$.

Then, we get

$$
\Delta g_{p}(x, y, t)=\frac{x^{p}}{p !} f^{(p, 0,0)}(0, y, t)+\sum_{j=0}^{n-2} \sum_{k=0}^{n-1-j} a_{k j}^{p} x^{p+2+2 k+2 j} \frac{\partial^{2 k}}{\partial x^{2 k}}\left[f^{(p, 0, j+1)}(0, y, t)\right]+O\left(x^{m}\right) .
$$

Noting that

$$
\sum_{j=0}^{n} \sum_{k=0}^{n-j} A_{k j}^{p}=\sum_{j=0}^{n} \sum_{k=0}^{n-1-j} A_{k j}^{p}+\sum_{j=0}^{n-1} A_{n-j, j}^{p}+A_{0 n}^{p} .
$$

Then, the proof of lemma is complete.

We now complete the proof of the theorem. In $\bar{\Omega}$ we define a function $\phi(x, y, t) \in C_{m+2+\alpha}(\bar{\Omega})$, that coincides on $x=0$ with $\psi(y, t)$ i.e.

$$
\left.\phi(x, y, t)\right|_{\Gamma_{0}}=\psi(y, t) .
$$

The function $V(x, y, t)=u(x, y, t)-\phi(x, y, t)$ satisfies in $\Omega$ the Dirichlet problem

$$
\begin{gathered}
\Delta V-V_{t}=g^{\star}(x, y, t), \\
V=\psi^{\star}(x, t) \text { on } \Gamma_{\omega}, \\
V=\phi_{1}(y, t)=0 \text { on } \Gamma_{\omega},
\end{gathered}
$$

where

$$
\begin{gathered}
R(x, y, t)=\Delta \phi-\phi_{t}, \\
g^{\star}(x, y, t)=f(x, y, t)-R(x, y, t), \\
\psi^{\star}(r, t)=\psi(r, t)-\xi(r, t) .
\end{gathered}
$$

Then $R \in C_{m+\alpha}(\bar{\Omega}), \quad \xi(0, t)=\psi(0, t)$, and form the compatibility condition (III), we get

$$
\xi_{\omega}^{(v q)}=L R-\sum_{k=0}^{\left[\frac{p}{2}\right]}(-1)^{v+k}\left(\begin{array}{c}
{\left[\frac{p}{2}\right]} \\
k
\end{array}\right) \phi_{k}^{0, v q-2 k} .
$$

Then the functions $g^{\star}(x, y, t), \quad \psi^{\star}$ and $\phi_{1}$ satisfy the compatibility conditions

$$
\psi_{\omega}^{\star(v q)}=L g^{\star} .
$$


Thus to prove the theorem, it is sufficient to consider the problem

$$
\Delta U-U_{t}=f(x, y, t),\left.\quad U\right|_{\Gamma_{\omega}}=\psi(r, t),\left.\quad U\right|_{\Gamma_{0}}
$$

where $f$ and $\psi$ satisfy the compatibility condition

$$
\psi_{\omega}^{(v q)}=L f
$$

consider the function $g(x, y, t) \in C_{m+2+\alpha}(\bar{\Omega})$ given by $g(x, y, t)=\sum_{p=0}^{m} g_{p}(x, y, t)$, where $g_{p}(x, y, t)$ are the function constructed in lemma 2. Now

$$
\begin{gathered}
\left(\Delta-\frac{\partial}{\partial t}\right)=R^{\star}, \\
R^{\star}(x, y, t)=\sum_{p=0}^{m}\left\{\frac{x^{p}}{p !} f^{(p, 0,0)}(0, y, t)+\sum_{j=0}^{n-1} \sum_{k=0}^{n-1-j} a_{k j}^{p} x^{p+2+2 k+2 j} \frac{\partial^{2 k}}{\partial x^{2 k}}\left[f^{(p, 0, j+1)}(0, y, t)\right.\right. \\
\left.\left.-f^{\star(p, 0, j+1)}(x, y, t)\right]\right\}+O\left(x^{m}\right) .
\end{gathered}
$$

Consider the function $V(x, y, t)=U(x, y, t)-g(x, y, t)$. This function satisfies in $\Omega$ the initial Dirichlet problem

$$
\begin{gathered}
\Delta V-V_{t}=h(x, y, t), \text { where } \\
h(x, y, t)=f(x, y, t)-R^{\star}(x, y, t), \\
V=\psi^{\star}(x, t)=\psi(x, t)-g(x, 0, t) \quad \text { on } \Gamma_{1} .
\end{gathered}
$$

Since for the function $g(x, y, t) \in C_{m+2+\alpha}(\bar{\Omega})$ we can prove that

$$
g_{\omega}^{(v q)}(0, t)=L R^{\star}-\sum_{k=0}^{\left[\frac{p}{2}\right]}(-1)^{v+k}\left(\begin{array}{c}
{\left[\frac{p}{2}\right]} \\
k
\end{array}\right) g_{k}^{0, v q-2 k}(0,0, t) .
$$

From $(11)$ and $g(x, y, t)=O\left(x^{2}\right)$, we have

$$
\begin{gathered}
g_{r}^{(0,2 v)}(0, y, t)=0, \quad v=1,2,3, \cdots, n+1, \quad r=0,1,2,3, \cdots, m+2, \quad \text { and } \\
\psi_{\omega}^{(v q)}(0, t)-g_{\omega}^{(0, v q)}(0,0, t)=L\left(f-R^{\star}\right)
\end{gathered}
$$

$h(x, y)$ and $\psi^{\star}$ satisfy the compatibility condition $(10)$, which may now be written in the form

$$
\psi_{\omega}^{\star^{(v q)}}(0, t)=0, \quad v=0,1, \cdots,\left[\frac{m+2+\alpha}{q}\right] .
$$


Consider now the sector of cylinder $\Omega$, bounded by the two planes $\left(Y_{1} \times J\right), Y_{1}=\{y: y=x \cot \omega\}$ and $\left(Y_{2} \times J\right), \quad Y_{2}=\{y: y=x \cot \omega\}$ and the surface $y=r(x, t)$. In $\Omega_{1}$ we define the functions $u_{1}(x, y, t)$ and $f_{1}(x, y, t)$ as follows

$$
\begin{aligned}
& u_{1}(x, y, t)= \begin{cases}V(x, y, t) & \text { if } \quad(x, y, t) \in \bar{\Omega} \\
-V(-x, y, t) & \text { if } \quad \notin \bar{\Omega},\end{cases} \\
& f_{1}(x, y)= \begin{cases}h(x, y, t) & \text { if } \quad(x, y, t) \in \bar{\Omega} \\
-h(-x, y, t) & \text { if } \quad \notin \bar{\Omega} .\end{cases}
\end{aligned}
$$

In $\Omega_{1}, \quad u_{1}(x, y, t) \in C_{2} \quad$ and $\quad f_{1}(x, y, t) \in C_{m+\alpha}(\bar{\Omega})$ and

$$
\Delta u=f_{1}(x, y, t)
$$




\section{References.}

1. Adams, R. A., Sobolev Spaces, Academic Press, New York, San Francisco, London, 1979.

2. Babus̃ka, I., Guo, B.Q., Regularity of the solution of elliptic problems with piecewise analytic data. Part I: Boundary value problems for linear elliptic equations of second order, SIAM J. Math. Anal. 19, 172203 (1988).

3. Babus̃ka, I., Guo, B.Q., Regularity of the solution of elliptic problems with piecewise analytic data, Part 2: The trace spaces and applications to the boundary value problems with nonhomogeneous boundary conditions, SIAM J. Math. Anal. 20, 763781 (1989).

4. Babus̃ka, I., Guo, B.Q., The $h-p$ version of the finite element method for problems with non-homogeneous essential boundary conditions, Comp. Meth. Appl. Mech. Engrg., 74, 128 (1989).

5. Babus̃ka, I., Guo, B.Q., Osborn, J., The regularity and numerical solutions of eigenvalue problems with piecewise analytic data, SIAM J. Numer. Anal. 25, 15341564 (1989).

6. Babus̃ka, I., Guo, B.Q., Stephan, E., On the exponential convergence of the $h-p$ version for boundary element Galerkin methods on polygons, Math. Meth. Appl. Sci., 12, 413-427 (1990).

7. Bergh, J., Lofstrom, J., Interpolation Spaces, An introduction, Springer-Verlag, Berlin, Heidelberg, New York, 1976.

8. Costabel, M., Dauge, M., General edge asymptotics solutions of second order elliptic boundary value problems I\&II, Proc. Roy. Soc. Edinburgh 123A, 109155, 157184 (1993).

9. Dauge, M., Elliptic Boundary Value Problems on Corner Domains, Lecture Notes in Math. 1341, Springer, New York (1988).

10. Dauge, M., Higher order oblique derivative problems on polyhedral domains , Comm. in PDE. 14, 11931227 (1989).

11. Friedman, A., Partial Di?erential Equations, Robert E. Krieger Publishing Company, Huntington, New York, 1976.

12. Grisvard, I., Singularities in Boundary Value Problems, Masson, Springer, Paris, Berlin (1992).

13. Grisvard, I., Elliptic Problems in Nonsmooth Domains, Pitman, Boston (1985).

14. Guo, B.Q., Babus̃ka, I., The $h-p$ version of the finite element method, Part 1: The basic approximation results, Comput. Mech. 1, 2141, (1986). 
15. Guo, B.Q., Babus̃ka, I., On the regularity of elasticity problems with piecewise analytic data, Adv. in Appl. Math. 14, 307347 (1993).

16. Guo, B.Q., Oh, H.S., The $h-p$ version of the finite element method for problems with nterfaces, Int. J. Numer. Meth. Engrg., 37, 17411762 (1994).

17. Guo, B.Q., Stephan, E., The $h-p$ Version of the coupling of ?nite element and boundary element method in polyhedral domains, preprint, 1994.

18. Kondrat'ev, V.A., Boundary value problems for elliptic equations in domain with conic or angular points, Trans. Moscow Math. Soc., 16, 227313 (1967).

19. Kozlov, V., Wendland, W. L., Goldberg, H., The behaviour of elastic fields and boundary integral Mellin techniques near conical points, preprint, 1994.

20. Lehman, R.S., Developments at an analytic corner of solutions of elliptic partial differential equations, J. Math. Mech. 8, 727760 (1984).

21. Lubuma, J. M.-S., Nicaise, S., Dirichlet problems in polyhedral domains, I: Regularity of the solutions, Math. Nachr., 168, 1994.

22. Maz'ya, V.G., Sobolev Spaces, Springer-Verlag, Berlin, Heidelberg, New York, Tokyo, 1985.

23. Maz'ya, V.G., Plamenevskii, B.A., On the coefficients in the asymptotic of solutions of elliptic boundary value problems in domain with conical points, Amer. Math. Soc. Transl. (2), Vol., 123,5788 (1984).

24. Morrey, C.B., Multiple Integrals in Calculus of Variations, Springer Verlag, New York, 1966.

25. Nicaise, S., Polygonal Interface Problems: Higher Regularity Results, Comm. in PDE. 15, 1475-1508 (1990).

26. Petersdorff, V.T., Boundary integral equations for mixed Dirichlet, Newumann and transmission conditions, Math. Meth. Appl. Sci., 11, 185213 (1989).

27. Schmutzler, B., Branching a symptotics for elliptic boundary value problems in a wedge, Boundary Value Problems and Integral Equations in Nonsmooth Domains, eds: M. Costabel, M. Dauge and S. Nicaise, 255267, Marcel Dekker Inc., (1994).

28. Stein, E.M., Singular Integral and Differentiability Properties of Functions, Princeton Univ. Press, Princeton Univ., New Jersey,1970 